\title{
On The Analytical Solution of Perturbative Nonlinear Schrodinger Equations with Real Initial and Homogeneous Boundary Conditions
}

\author{
Maha A. El-Hazmy*
}

Girls College, Mathematics Department, Medina, P.O.B. 3193, Saudi Arabia

\begin{abstract}
In this paper, a perturbing nonlinear Schrodinger equation is studied under limited time interval through homogeneous boundary conditions and real initial condition. The analytical solution for the linear case is introduced. The perturbation method is used to introduce an approximate solution for the perturbative nonlinear case for which a power series solution is proved to exist. Using Mathematica, the solution algorithm is tested through first order approximation. The method of solution is illustrated through case studies and figures.
\end{abstract}

Keywords: Nonlinear Schrodinger equation, Perturbation, Eigenfunction expansion.

\section{INTRODUCTION}

In many fields such as plasma physics [1], quantum mechanics [2] and wave propagation in nonlinear media [3,4], the nonlinear Schrodinger equation (NLS) is the principal equation to be analyzed and solved. In the literature, there are a lot of NLS problems depending on additive or multiplicative noise in the random case $[5,6]$ or a lot of solution methodologies in the deterministic case.

Wang et al. [7] obtained the exact solutions to NLS using what they called the sub-equation method. They got four kinds of exact solutions of the equation

$i \frac{\partial u}{\partial t}+\frac{1}{2} \frac{\partial^{2} u}{\partial x^{2}}+\alpha|u|^{p} u+\beta|u|^{2 p} u=0$,

for which no sign to the initial or boundary conditions type is made. Xu L. and Zhang J. [8] followed the same previous technique in solving the higher order NLS:

$$
\begin{gathered}
i \frac{\partial u}{\partial x}-\frac{1}{2} \alpha \frac{\partial^{2} u}{\partial t^{2}}+\beta|u|^{2} u+i \varepsilon \frac{\partial^{3} u}{\partial t^{3}}+. \\
i \delta|u|^{2} \frac{\partial u}{\partial t}+i \gamma u^{2} \frac{\partial u^{*}}{\partial t}=0
\end{gathered}
$$

Sweilam [9] solved

$i \frac{\partial u}{\partial t}+\frac{\partial^{2} u}{\partial x^{2}}+q|u|^{2} u=0, t>0, L_{0}<x<L_{1}$,

with initial condition $u(x, 0)=g(x)$ and boundary conditions $u_{x}\left(L_{0}, t\right)=u_{x}\left(L_{1}, t\right)=0$ which gives rise to solitary solutions using variational iteration method. Zhu [10] used the extended hyperbolic auxiliary equation method in getting the exact explicit solutions to the higher order NLS:

$i q_{z}-\frac{\beta_{1}}{2} q_{t t}+\gamma_{1}|q|^{2} q=i \frac{\beta_{2}}{6} q_{t t t}+\frac{\beta_{3}}{24} q_{t t t t}-\gamma_{2}|q|^{4} q$,

*Address correspondence to this author at the Girls college, Mathematics Department, Medina, P.O.B. 3193; Saudi Arabia;

E-mail: moon14212@hotmail.com without any conditions. Sun et al. [11] solved the NLS:

$$
i \frac{\partial \psi}{\partial t}+\frac{\partial^{2} \psi}{\partial x^{2}}+a|\psi|^{2} \psi=0
$$

with the initial condition $\psi(x, 0)=\psi_{0}(x)$ using Lie group method. By using coupled amplitude phase formulation, Parsezian and Kalithasan [12] constructed the quartic anharmonic oscillator equation from the coupled higher order NLS. Two-dimensional grey solitons to the NLS were numerically analyzed by Sakaguchi and Higashiuchi [13]. The generalized derivative NLS was studied by Huang et al. [14] introducing a new auxiliary equation expansion method. ElTawil et al. introduced a new approach in solving a perturbative cubic nonlinear Schrodinger equation in [15] and with variable group velocity in [16].

In this paper, a straight forward solution algorithm is introduced using the transformation from a complex solution to coupled equations in two real solutions. Eliminating one of the real solutions to get separate independent equations, a higher order equation is obtained in each variable. Finally, a perturbative approximate solution to the system is introduced. Section 2 illustrates the method applied on the linear equation. Section 3 deals with the nonlinear case.

\section{THE LINEAR CASE} tion:

Consider the non homogeneous linear Schrodinger equa-

$$
\begin{aligned}
& i \frac{\partial u(t, z)}{\partial z}+\alpha \frac{\partial^{2} u(t, z)}{\partial t^{2}}+i \gamma u(t, z) \\
& \quad=F(t, z),(t, z) \in(0, \zeta) \times(0, \infty)
\end{aligned}
$$

where $u(t, z)$ is a complex valued function which is subjected to:

I.C.: $u(t, 0)=f(t)$, a real valued function,

$B C .: u(0, z)=0, u(\zeta, z)=0$. 
Let $u(t, z)=\psi(t, z)+i \phi(t, z), \quad \psi, \phi$ : real valued functions. The following coupled equations are obtained:

$\frac{\partial \phi(t, z)}{\partial z}=\alpha \frac{\partial^{2} \psi(t, z)}{\partial t^{2}}-\gamma \phi(t, z)-F(t, z)$,

$\frac{\partial \psi(t, z)}{\partial z}=-\alpha \frac{\partial^{2} \phi(t, z)}{\partial t^{2}}-\gamma \psi(t, z)$,

where $\psi(t, 0)=f(t)$ while all corresponding other I.C. and B.C. are zeros.

To get rid of the loss terms in equations (4) and (5), one can use the following effective transformations:

$\psi(t, z)=e^{-\gamma z} w(t, z)$,

$\phi(t, z)=e^{-\gamma z} v(t, z)$,

where $w(t, 0)=f(t)$ while all corresponding other I.C. and B.C. are zeros. The following coupled equations are obtained:

$\frac{\partial v(t, z)}{\partial z}=\alpha \frac{\partial^{2} w(t, z)}{\partial t^{2}}-G_{1}(t, z)$,

$\frac{\partial w(t, z)}{\partial z}=-\alpha \frac{\partial^{2} v(t, z)}{\partial t^{2}}$,

where

$G_{1}(t, z)=e^{\gamma z} F(t, z)$.

Eliminating one of the variables in equations (8) and (9), one can get the following independent equations:

$\frac{\partial^{4} w(t, z)}{\partial t^{4}}+\frac{1}{\alpha^{2}} \frac{\partial^{2} w(t, z)}{\partial z^{2}}=\frac{1}{\alpha^{2}} \tilde{\psi}_{1}(t, z)$,

$\frac{\partial^{4} v(t, z)}{\partial t^{4}}+\frac{1}{\alpha^{2}} \frac{\partial^{2} v(t, z)}{\partial z^{2}}=\frac{1}{\alpha^{2}} \tilde{\psi}_{2}(t, z)$,

where

$\tilde{\psi}_{1}(t, z)=\alpha \frac{\partial^{2} G_{1}(t, z)}{\partial t^{2}}$,

$\tilde{\psi}_{2}(t, z)=-\frac{\partial G_{1}(t, z)}{\partial z}$

Using the eigenfunction expansion technique [17], the following solution expressions are obtained:

$w(t, z)=\sum_{n=0}^{\infty} T_{n}(z) \sin \left(\frac{n \pi}{T}\right) t$,

$v(t, z)=\sum_{n=0}^{\infty} \tau_{n}(z) \sin \left(\frac{n \pi}{T}\right) t$,

where $T_{n}(z)$ and $\tau_{n}(z)$ can be got through the applications of initial conditions and then solving the resultant second order differential equations using the method of the variational parameter [18]. The final expressions can be got as the following:

$T_{n}(z)=A_{1}(z) \sin \beta_{n} z+\left(C_{2}+B_{1}(z)\right) \cos \beta_{n} z$,

$\tau_{n}(z)=A_{2}(z) \sin \beta_{n} z+\left(\tilde{C}_{2}+B_{2}(z)\right) \cos \beta_{n} z$,

where

$\beta_{n}=\alpha\left(\frac{n \pi}{T}\right)^{2}$,

$A_{1}(z)=\frac{1}{\beta_{n}} \int \tilde{\psi}_{1 n}(z ; n) \cos \left(\beta_{n}\right) z d z$,

$B_{1}(z)=\frac{-1}{\beta_{n}} \int \tilde{\psi}_{1 n}(z ; n) \sin \left(\beta_{n}\right) z d z$,

$A_{2}(z)=\frac{1}{\beta_{n}} \int \tilde{\psi}_{2 n}(z ; n) \cos \left(\beta_{n}\right) z d z$,

$B_{2}(z)=\frac{-1}{\beta_{n}} \int \tilde{\psi}_{2 n}(z ; n) \sin \left(\beta_{n}\right) z d z$,

in which

$\tilde{\psi}_{1 n}(z ; n)=\frac{2}{T} \int_{0}^{T} \tilde{\psi}_{1}(t, z) \sin \left(\frac{n \pi}{T}\right) t d t$,

$\tilde{\psi}_{2 n}(z ; n)=\frac{2}{T} \int_{0}^{T} \tilde{\psi}_{2}(t, z) \sin \left(\frac{n \pi}{T}\right) t d t$.

The following conditions should also be satisfied:

$C_{2}=\frac{2}{T} \int_{0}^{T} f(t) \sin \left(\frac{n \pi}{T}\right) t d t-B_{1}(0)$,

$\tilde{C}_{2}=-B_{2}(0)$.

Finally, the following solution is obtained:

$u(t, z)=e^{-\gamma z}(w(t, z)+i v(t, z))$,

or

$|u(t, z)|^{2}=e^{-2 \gamma z}\left(w^{2}(t, z)+v^{2}(t, z)\right)$.

\section{Example-1}

Solving equations (1), (2) and (3) with taking $f(t)=\rho$, $F(t, z)=0$ and following the previous solution algorithm, the following final result is obtained:

$u(t, z)=\frac{2 \rho}{\pi} e^{-\gamma_{z}} \sum_{n=1}^{\infty} \frac{\left[1-(-1)^{n}\right]}{n} \cos \beta_{n} z \sin \left(\frac{n \pi}{T}\right) t$.

One can justify the following facts:

i) $\lim _{\rho \rightarrow 0} u(t, z)=0$,

ii) $\lim _{\gamma \rightarrow \infty} u(t, z)=0, z>0$, 
iii) $\lim _{\alpha \rightarrow 0} u(t, z)=\rho e^{-\gamma z}$,

iv) $\lim _{z \rightarrow \infty} u(t, z)=0, \gamma>0$,

which coincide with the physical conditions. The following figures illustrate some cases:

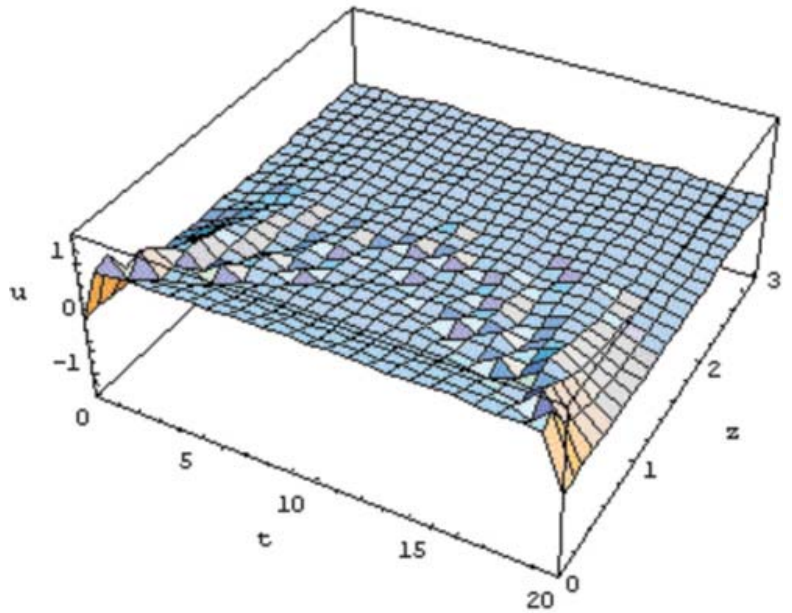

Fig. (1). The solution $\mathrm{u}$ at $\alpha, \gamma, \rho=1, T=20$ for 50 terms using mathematica 5 .

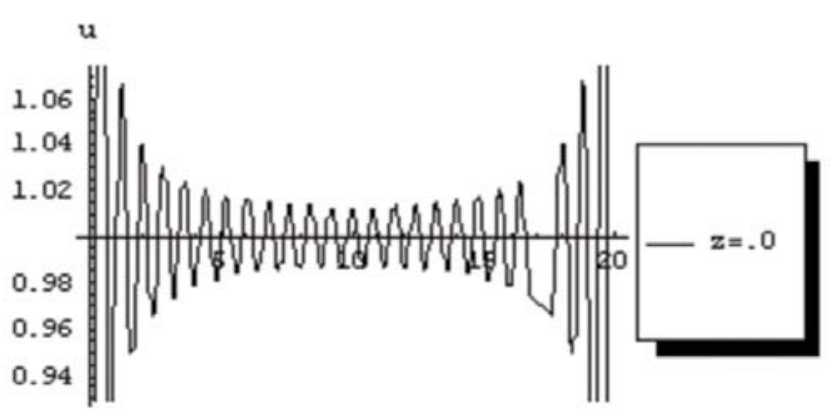

Fig. (2). The solution $\mathrm{u}$ at $\mathrm{z}=0$ and $\alpha, \gamma, \rho=1, T=20$ for 50 terms.

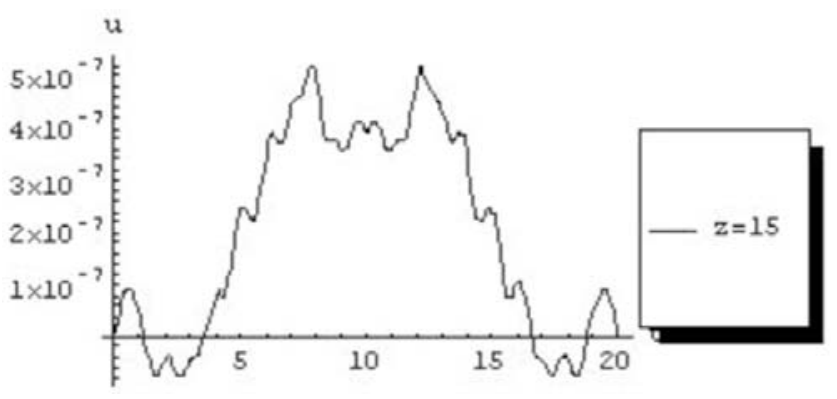

Fig. (3). The solution $\mathrm{u}$ at $\mathrm{z}=15$ and $\alpha, \gamma, \rho=1, T=20$ for 50 terms.

One can notice the high reduction of the solution level.

\section{Example-2}

Taking the case of $f(t)=\rho \sin \left(\frac{m \pi}{T}\right) t$, the following final result is obtained: $u(t, z)=\rho e^{-\gamma z} \cos \beta_{m} z \sin \left(\frac{m \pi}{T}\right) t$,

where

$\beta_{m}=\alpha\left(\frac{m \pi}{T}\right)^{2}$.

The following figures illustrate the solution at different cases:

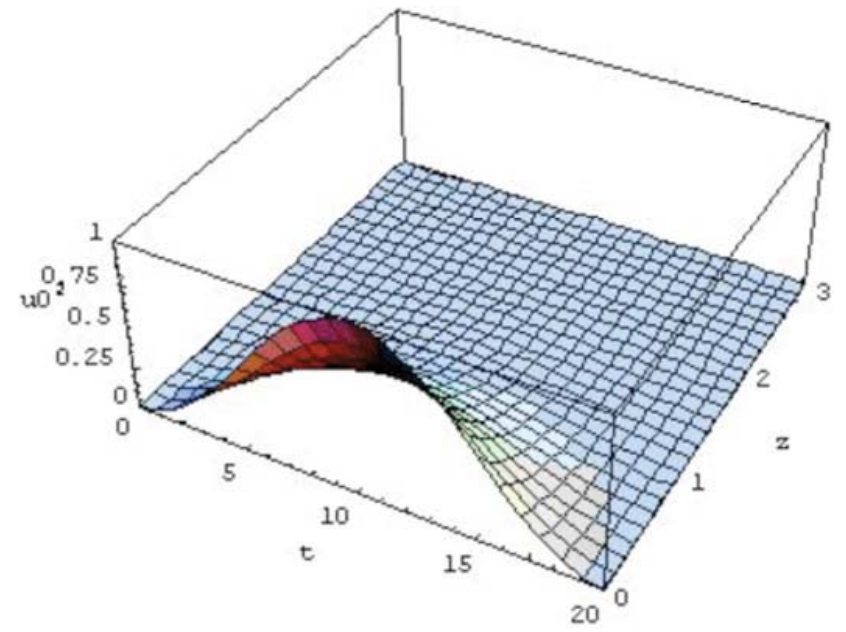

Fig. (4). The solution $|u|^{2}$ at $\alpha, \gamma, \rho, m=1, T=20$.

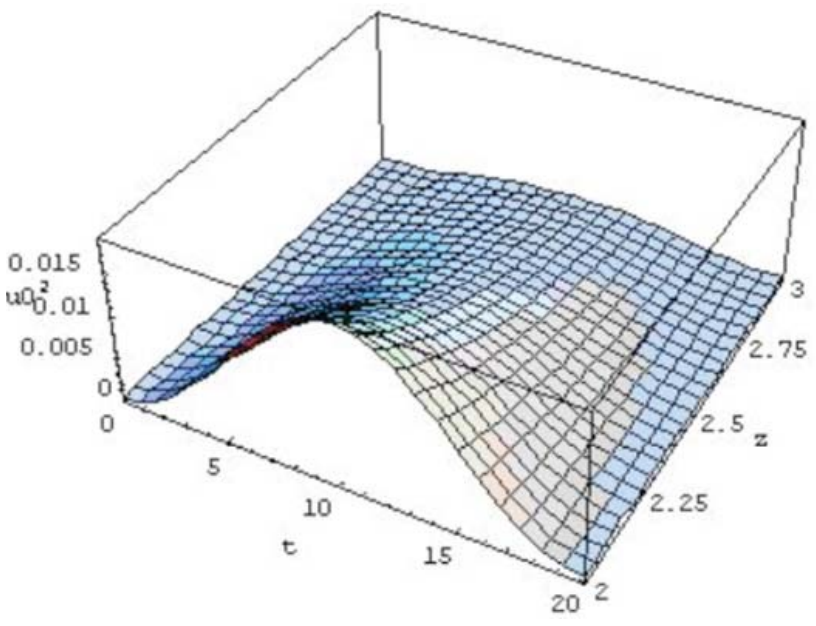

Fig. (5). The solution $|u|^{2}$ at $\alpha, \gamma, \rho, m=1, T=20$ for different $z$ range.

One can also notice the high reduction of the solution level.

\section{THE NON- LINEAR CASE}

Consider the homogeneous non-linear Schrodinger equation:

$$
\begin{gathered}
i \frac{\partial u(t, z)}{\partial z}+\alpha \frac{\partial^{2} u(t, z)}{\partial t^{2}}+\varepsilon|u(t, z)|^{2} u(t, z)+ \\
i \gamma u(t, z)=0,(t, z) \in(0, \zeta) \times(0, \infty)
\end{gathered}
$$


where $u(t, z)$ is a complex valued function which is subjected to the initial and boundary conditions (2) and (3).

\section{Lemma}

The solution of equation (31) with the constraints (2) and (3) is a power series in $\varepsilon$ if exists.

\section{Proof}

At $\varepsilon=0$, the following linear equation is got:

$$
\begin{gathered}
i \frac{\partial u(t, z)}{\partial z}+\alpha \frac{\partial^{2} u(t, z)}{\partial t^{2}}+i \gamma u(t, z) \\
=0,(t, z) \in(0, \zeta) \times(0, \infty)
\end{gathered}
$$

which has the solution

$u_{0}(t, z)=e^{-\gamma z}\left(w_{0}(t, z)+i v_{0}(t, z)\right)$,

where

$$
\begin{aligned}
& w_{0}(t, z)=\sum_{n=0}^{\infty} A_{n} \cos \beta_{n} z \sin \left(\frac{n \pi}{T}\right) t, \\
& v_{0}(t, z)=\sum_{n=0}^{\infty} B_{n} \sin \beta_{n} z \sin \left(\frac{n \pi}{T}\right) t,
\end{aligned}
$$

in which

$$
A_{n}=\frac{2}{T} \int_{0}^{T} f(t) \sin \left(\frac{n \pi}{T}\right) t d t
$$

$B_{n}:$ arbitrary constant.

Following Pickard approximation, equation (31) can be rewritten as

$$
\begin{gathered}
i \frac{\partial u_{n}(t, z)}{\partial z}+\alpha \frac{\partial^{2} u_{n}(t, z)}{\partial t^{2}}+i \gamma u_{n}(t, z) \\
=-\varepsilon\left|u_{n-1}(t, z)\right|^{2} u_{n-1}(t, z), n \geq 1 .
\end{gathered}
$$

At $n=1$, the iterative equation takes the following form:

$$
\begin{gathered}
i \frac{\partial u_{1}(t, z)}{\partial z}+\alpha \frac{\partial^{2} u_{1}(t, z)}{\partial t^{2}}+i \gamma u_{1}(t, z)= \\
-\varepsilon\left|u_{0}(t, z)\right|^{2} u_{0}(t, z)=\varepsilon h_{1}(t, z)
\end{gathered}
$$

which can be solved as a linear case with zero initial and boundary conditions. The following general solution can be obtained:

$$
\begin{aligned}
w_{1}(t, z) & =\sum_{n=0}^{\infty}\left(T_{0 n}+\varepsilon T_{1 n}\right) \sin \left(\frac{n \pi}{T}\right) t \\
v_{1}(t, z) & =\sum_{n=0}^{\infty}\left(\tau_{0 n}+\varepsilon \tau_{1 n}\right) \sin \left(\frac{n \pi}{T}\right) t \\
u_{1}(t, z) & =e^{-\gamma z}\left(w_{1}(t, z)+i v_{1}(t, z)\right) \\
& =u_{1}^{(0)}+\varepsilon u_{1}^{(1)}
\end{aligned}
$$

At $n=2$, the following equation is obtained:

$$
\begin{gathered}
i \frac{\partial u_{2}(t, z)}{\partial z}+\alpha \frac{\partial^{2} u_{2}(t, z)}{\partial t^{2}}+i \gamma u_{2}(t, z)= \\
-\varepsilon\left|u_{1}(t, z)\right|^{2} u_{1}(t, z)=\varepsilon h_{2}(t, z)
\end{gathered}
$$

which can be solved as a linear case with zero initial and boundary conditions. The following general solution can be obtained:

$u_{2}(t, z)=u_{2}^{(0)}+\varepsilon u_{2}^{(1)}+\varepsilon^{2} u_{2}^{(2)}+\varepsilon^{3} u_{2}^{(3)}+\varepsilon^{4} u_{2}^{(4)}$.

Continuing like this, one can get

$$
\begin{aligned}
& u_{n}(t, z)=u_{n}^{(0)}+\varepsilon u_{n}^{(1)}+\varepsilon^{2} u_{n}^{(2)}+\varepsilon^{3} u_{n}^{(3)}+ \\
& \ldots \ldots \ldots+\varepsilon^{(n+m)} u_{n}^{(n+m)}
\end{aligned}
$$

As $n \rightarrow \infty$, the solution (if exists) can be reached as $u(t, z)=\lim _{n \rightarrow \infty} u_{n}(t, z)$. Accordingly, the solution is a power series in $\varepsilon . / / / /$

According to the previous lemma, one can assume the solution of equation (31) as the following:

$u(t, z)=\sum_{n=0}^{\infty} \varepsilon^{n} u_{n}$

Let $u(t, z)=\psi(t, z)+i \phi(t, z), \quad \psi, \phi$ : real valued functions. The following coupled equations are got:

$$
\begin{aligned}
& \frac{\partial \phi(t, z)}{\partial z}=\alpha \frac{\partial^{2} \psi(t, z)}{\partial t^{2}}+\varepsilon\left(\psi^{2}+\phi^{2}\right)-\gamma \phi(t, z), \\
& \frac{\partial \psi(t, z)}{\partial z}=-\alpha \frac{\partial^{2} \phi(t, z)}{\partial t^{2}} \\
& -\varepsilon\left(\psi^{2}+\phi^{2}\right)-\gamma \psi(t, z),
\end{aligned}
$$

where $\psi(t, 0)=f(t)$ and all corresponding other I.C. and B.C. are zeros.

As a second order perturbation solution, one can assume that

$$
\begin{aligned}
& \psi(t, z)=\psi_{0}+\varepsilon \psi_{1}+\varepsilon^{2} \psi_{2}, \\
& \phi(t, z)=\phi_{0}+\varepsilon \phi_{1}+\varepsilon^{2} \phi_{2},
\end{aligned}
$$

where $\psi_{0}(t, 0)=f(t)$ and all corresponding other I.C. and B.C. are zeros.

Substituting from equations (35) and (36) into equations (33) and (34) and then equating the equal powers of $\varepsilon$, one can get the following set of coupled equations:

$$
\begin{aligned}
& \frac{\partial \phi_{0}(t, z)}{\partial z}=\alpha \frac{\partial^{2} \psi_{0}(t, z)}{\partial t^{2}}-\gamma \phi_{0}(t, z), \\
& \frac{\partial \psi_{0}(t, z)}{\partial z}=-\alpha \frac{\partial^{2} \phi_{0}(t, z)}{\partial t^{2}}-\gamma \psi_{0}(t, z), \\
& \frac{\partial \phi_{1}(t, z)}{\partial z}=\alpha \frac{\partial^{2} \psi_{1}(t, z)}{\partial t^{2}}-\gamma \phi_{1}(t, z)+\left(\psi_{0}^{3}+\psi_{0} \phi_{0}^{2}\right),
\end{aligned}
$$




$$
\begin{aligned}
& \frac{\partial \psi_{1}(t, z)}{\partial z}=-\alpha \frac{\partial^{2} \phi_{1}(t, z)}{\partial t^{2}}-\gamma \psi_{1}(t, z)-\left(\phi_{0}^{3}+\phi_{0} \psi_{0}^{2}\right), \\
& \frac{\partial \phi_{2}(t, z)}{\partial z}=\alpha \frac{\partial^{2} \psi_{2}(t, z)}{\partial t^{2}}-\gamma \phi_{2}(t, z) \\
& \quad+\left(3 \psi_{0}^{2} \psi_{1}+2 \psi_{0} \phi_{0} \phi_{1}+\psi_{1} \phi_{0}^{2}\right), \\
& \frac{\partial \psi_{2}(t, z)}{\partial z}=-\alpha \frac{\partial^{2} \phi_{2}(t, z)}{\partial t^{2}}-\gamma \psi_{2}(t, z) . \\
& -\left(3 \phi_{0}^{2} \phi_{1}+2 \phi_{0} \psi_{0} \psi_{1}+\phi_{1} \psi_{0}^{2}\right)
\end{aligned}
$$

Following the solution algorithm described in the previous section for the linear case, the following final results are obtained:

$u_{0}(t, z)=e^{-\gamma z}\left(w_{0}+i v_{0}\right)$,

$w_{0}(t, z)=\sum_{n=0}^{\infty}\left[A_{1} \sin \beta_{n} z+A_{2} \cos \beta_{n} z\right] \sin \left(\frac{n \pi}{T}\right) t$,

$v_{0}(t, z)=0$,

$A_{2}=\frac{2}{T} \int_{0}^{T} f(t) \sin \left(\frac{n \pi}{T}\right) t d t$.

Also,

$u_{1}(t, z)=e^{-\gamma z}\left(w_{1}+i v_{1}\right)$,

where

$w_{1}(t, z)=\sum_{n=0}^{\infty} T_{1 n}(z) \sin \left(\frac{n \pi}{T}\right) t$,

$v_{1}(t, z)=\sum_{n=0}^{\infty} \tau_{1 n}(z) \sin \left(\frac{n \pi}{T}\right) t$,

in which

$T_{1 n}(z)=A_{11}(z) \sin \beta_{n} z+\left(C_{12}+B_{11}(z)\right) \cos \beta_{n} z$,

$\tau_{1 n}(z)=A_{12}(z) \sin \beta_{n} z+\left(\tilde{C}_{12}+B_{12}(z)\right) \cos \beta_{n} z$,

where the constants and variables $A_{11}(z), C_{12}, B_{11}(z)$, $A_{12}(z), \tilde{C}_{12}$ and $B_{12}(z)$ can be evaluated in a similar manner as the corresponding ones in the linear case.

The final results are

$u_{2}(t, z)=e^{-\gamma z}\left(w_{2}+i v_{2}\right)$,

where

$$
\begin{aligned}
& w_{2}(t, z)=\sum_{n=0}^{\infty} T_{2 n}(z) \sin \left(\frac{n \pi}{T}\right) t, \\
& v_{2}(t, z)=\sum_{n=0}^{\infty} \tau_{2 n}(z) \sin \left(\frac{n \pi}{T}\right) t,
\end{aligned}
$$

in which

$T_{2 n}(z)=A_{21}(z) \sin \beta_{n} z+\left(C_{22}+B_{21}(z)\right) \cos \beta_{n} z$,

$\tau_{2 n}(z)=A_{22}(z) \sin \beta_{n} z+\left(\tilde{C}_{22}+B_{22}(z)\right) \cos \beta_{n} z$,

where the constants and variables $A_{21}(z), C_{22}, B_{21}(z)$, $A_{22}(z), \tilde{C}_{22}$ and $B_{22}(z)$ can be evaluated in a similar manner as the corresponding ones in the linear case.

\section{First and second order approximations}

In this case, the solution of equation (31) takes the following form

$u^{(1)}=u_{0}+\varepsilon u_{1}$.

Substituting from equations (43) and (47), one can get the following final expression:

$\left|u^{(1)}\right|^{2}=e^{-2 \gamma z}\left(w_{0}^{2}+2 \varepsilon w_{0} w_{1}+\varepsilon^{2}\left(w_{1}^{2}+v_{1}^{2}\right)\right)$.

The second order approximation takes the following form

$u^{(2)}=u_{0}+\varepsilon u_{1}+\varepsilon^{2} u_{2}$,

or

$$
\begin{aligned}
\left|u^{(2)}\right|^{2}=e^{-2 \gamma z}\left(w_{0}^{2}+2 \varepsilon w_{0} w_{1}+\right. & \\
& \varepsilon^{2}\left(w_{1}^{2}+v_{1}^{2}+2 w_{0} w_{2}\right)+ \\
& \varepsilon^{3}\left(2 w_{1} w_{2}+2 v_{1} v_{2}\right)+ \\
& \left.\varepsilon^{4}\left(w_{2}^{2}+v_{2}^{2}\right)\right)
\end{aligned}
$$

\section{Example-3}

Solving equations (31), (2) and (3) with taking $f(t)=\rho$ and following the previous solution algorithm, the following selective results for the first order approximation are obtained:

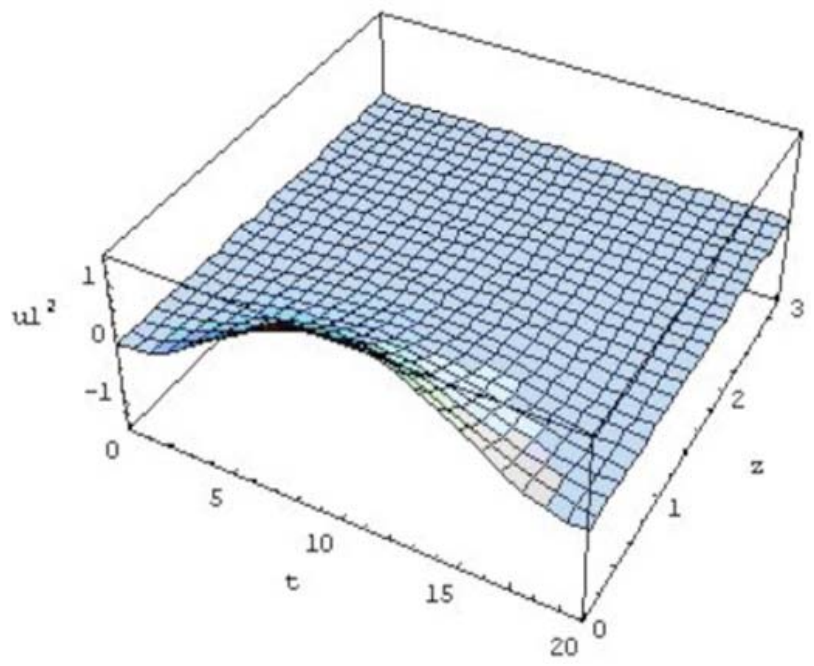

Fig. (6). The first order approximation of $\left|u^{(1)}\right|^{2}$ at $\varepsilon=0$ and $\alpha, \gamma, \rho=1, T=20$ and

only one term in the series $(\mathrm{m}=1)$. 


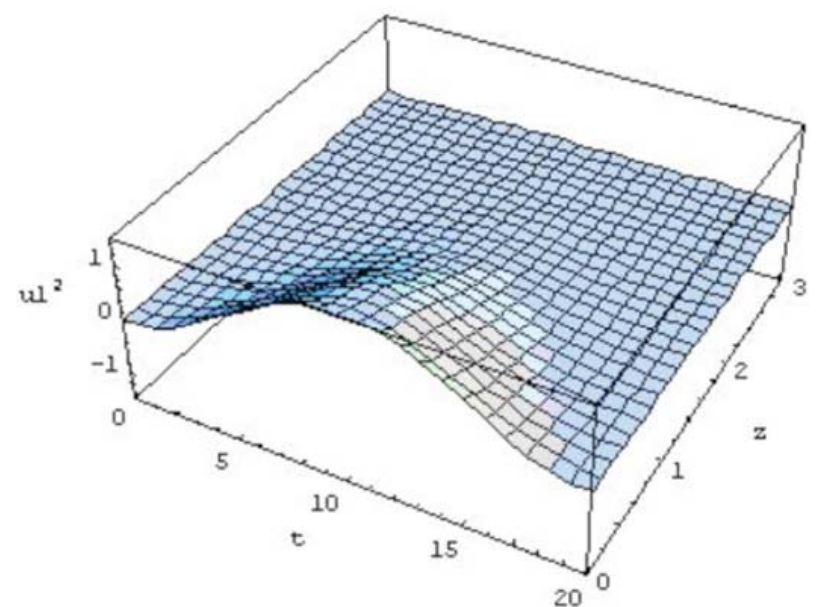

Fig. (7). The first order approximation of $\left|u^{(1)}\right|^{2}$ at $\varepsilon=2$ and $\alpha, \gamma, \rho=1, T=20$ and

only one term in the series $(\mathrm{m}=1)$.

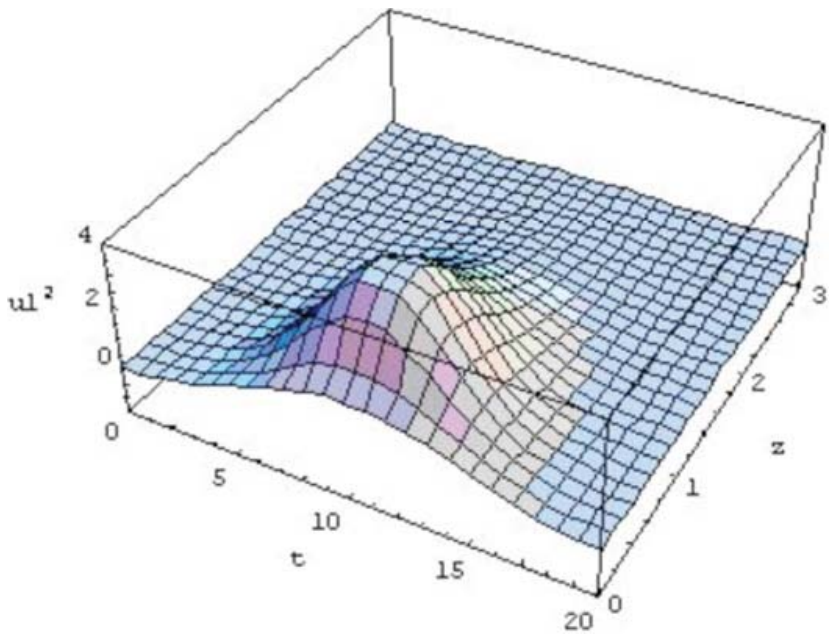

Fig. (8). The first order approximation of $\left|u^{(1)}\right|^{2}$ at $\varepsilon=5$ and $\alpha, \gamma, \rho=1, T=20$ and

only one term in the series $(\mathrm{m}=1)$.

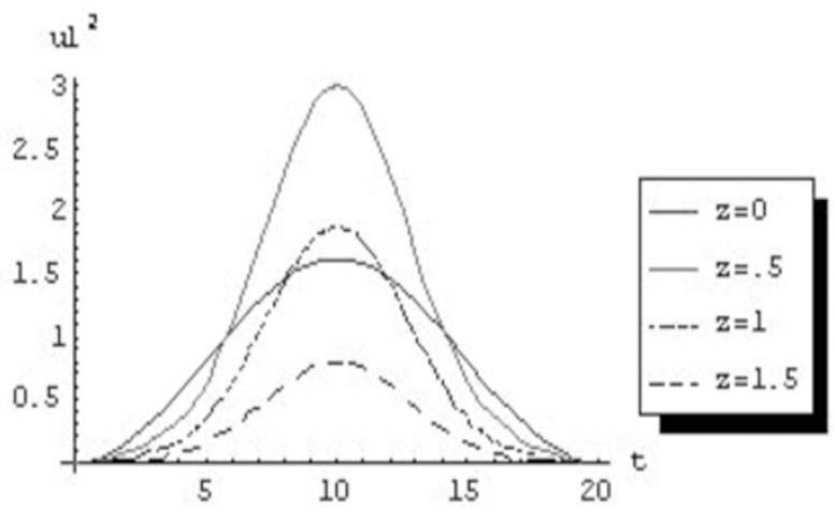

Fig. (9). The first order approximation of $\left|u^{(1)}\right|^{2}$ at $\varepsilon=4$ and $\alpha, \gamma, \rho=1, T=20$ and only one term in the series $(\mathrm{m}=1)$ for different $\mathrm{z}$ values.

\section{Example-4}

Taking the case of $f(t)=\rho \sin \left(\frac{m \pi}{T}\right) t$, the following final results for the first order approximation are obtained:

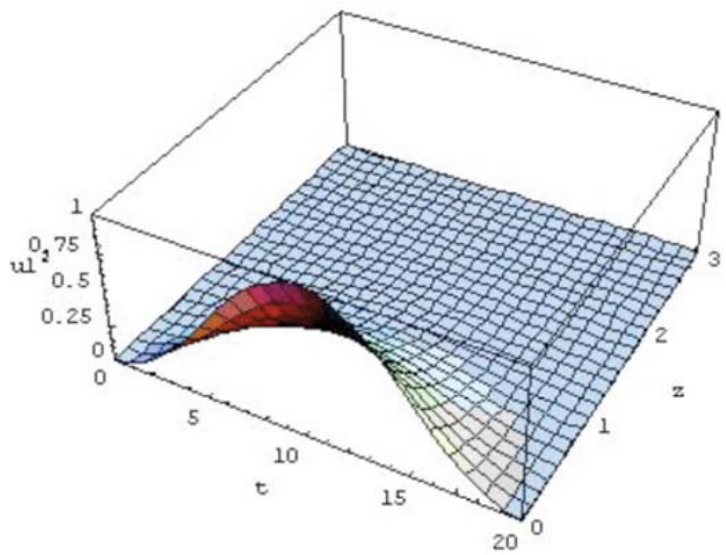

Fig. (10). The first order approximation of $\left|u^{(1)}\right|^{2}$ at $\varepsilon=0$ and $\alpha, \gamma, \rho=1, T=20(\mathrm{~m}=1)$.

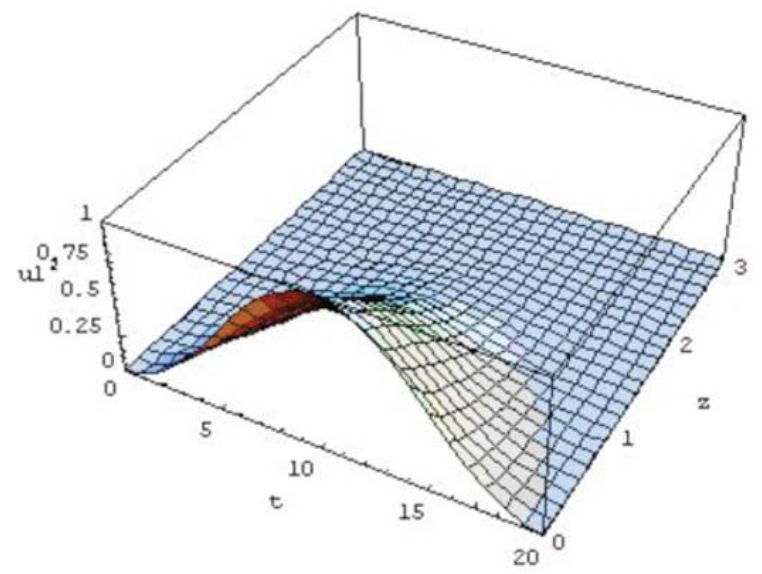

Fig. (11). The first order approximation of $\left|u^{(1)}\right|^{2}$ at $\varepsilon=3$ and $\alpha, \gamma, \rho=1, T=20(\mathrm{~m}=1)$.

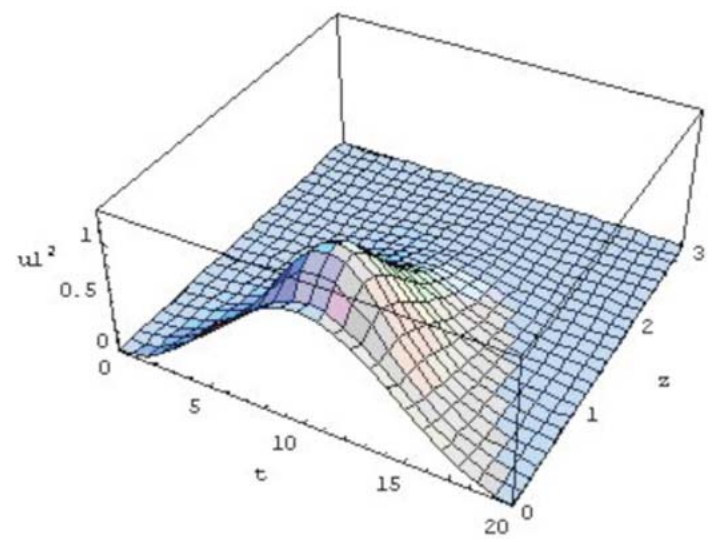

Fig. (12). The first order approximation of $\left|u^{(1)}\right|^{2}$ at $\varepsilon=5$ and $\alpha, \gamma, \rho=1, T=20(\mathrm{~m}=1)$. 


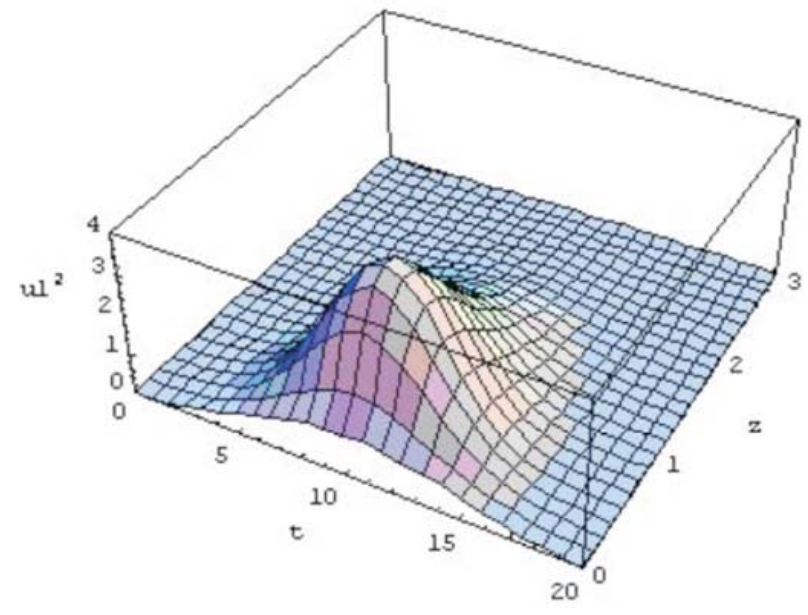

Fig. (13). The first order approximation of $\left|u^{(1)}\right|^{2}$ at $\varepsilon=3$ and $\alpha, \gamma, \rho=1, T=20(\mathrm{~m}=1)$.

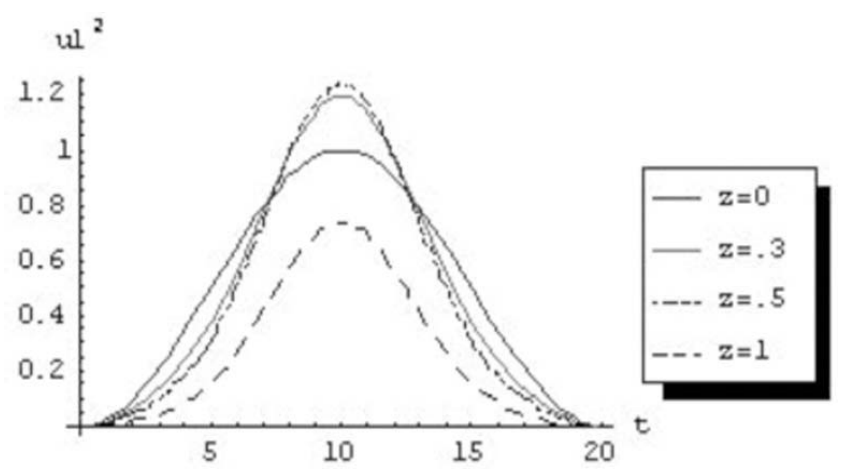

Fig. (14). The first order approximation of $\left|u^{(1)}\right|^{2}$ at $\varepsilon=5$ and $\alpha, \gamma, \rho=1, T=20(\mathrm{~m}=1)$ for different values of $\mathrm{z}$.

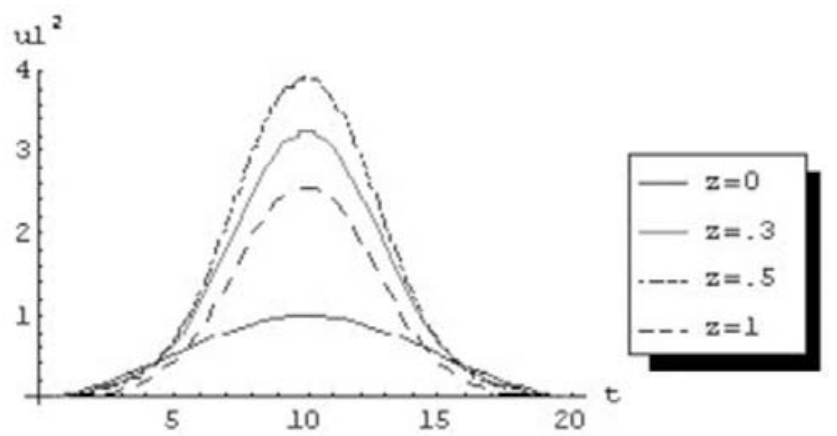

Fig. (15). The first order approximation of $\left|u^{(1)}\right|^{2}$ at $\varepsilon=10$ and $\alpha, \gamma, \rho=1, T=20(\mathrm{~m}=1)$ for different values of $\mathrm{z}$.

\section{CONCLUSIONS}

The perturbation technique introduces an approximate solution to the NLS equation with a perturbative nonlinear term under homogeneous boundary conditions and real initial condition for a finite interval. Using mathematica, the difficult and huge computations problems were fronted to some extent, even for the first order approximation and for limited series terms. To get more improved orders, it is expected to face a problem of computation. In general, the solution is decreased with the distance $\mathrm{z}$ and is greatly affected according to the initial pulse. The solution level is increased with the increase of the nonlinearity level.

\section{REFERENCES}

[1] Bruneau C, Menza L, Lehner T. Numerical resolution of some nonlinear Schrodinger-like equations in plasmas. Numer Meth PDEs 1999; 15(6): 672-96.

[2] Abdullaev F, Garnier J. Solitons in media with random dispersive perturbations. Physica (D) 1999; 134: 303-15.

[3] Faris WG, Tsay WJ. Time delay in random scattering. SIAM J Appl Math 1994; 54(2): 443-55.

[4] Cazenave T, Lions P. Orbital stability of standing waves for some nonlinear Schrodinger equations. Commun Math Phys 1982; 85: 549-61.

[5] Debussche A, Menza L. Numerical simulation of focusing stochastic nonlinear Schrodinger equations. Physica D 2002; 162: 131-54.

[6] Debussche A, Menza L. Numerical resolution of stochastic focusing NLS equations. Appl Math Lett 2002;15: 661-9.

[7] Wang M, and et al. Various exact solutions of nonlinear Schrodinger equation with two nonlinear terms. Chaos Solitons Fractals 2007; 31: 594-601.

[8] $\mathrm{Xu} \mathrm{L}$, Zhang J. Exact solutions to two higher order nonlinear Schrodinger equations. Chaos, Solitons Fractals 2007; 31: 937-42.

[9] Sweilam N. Variational iteration method for solving cubic nonlinear Schrodinger equation. J Comput Appl Math (submitted).

[10] Zhu S. Exact solutions for the high order dispersive cubic quintic nonlinear Schrodinger equation by the extended hyperbolic auxiliary equation method. Chaos Solitons Fractals (submitted).

[11] Sun J, and et al. New conservation schemes for the nonlinear Schrodinger equation. Appl Math Comput 2006; 177: 446-51.

[12] Parsezian K, Kalithasan B. Cnoidal and solitary wave solutions of the coupled higher order nonlinear Schrodinger equations in nonlinear optics. Chaos Solitons Fractals 2007; 31: 188-96.

[13] Sakaguchi H, Higashiuchi T. Two dimensional dark soliton in the nonlinear Schrodinger equation. Phys Lett A (submitted).

[14] Huang D, and et al. Explicit and exact traveling wave solution for the generalized derivative Schrodinger equation. Chaos Solitons Fractals 2007; 31: 586-93.

[15] El-Tawil M, El-Hazmy M. On perturbative cubic nonlinear Schrodinger equations. Far East J Appl Math 2007; 28(2): 323-39.

[16] El-Tawil M, El-Hazmy M. Perturbative nonlinear Schrodinger equations under variable group velocity dissipation. Far East J Math Sci 2007; 27(2): 415-30.

[17] Farlow S. P.D.E. for scientists and engineers. New York: John Wiley \& sons 1982.

[18] Pipes L, Harvill L. Applied mathematics for engineers and physicists. Tokyo: McGraw-Hill1970. 